Original Research Paper

\title{
Metabolic Features of Heart Failure with Different Etiology
}

\author{
Oleynikov Dmitrij Arkadievich \\ Department of Therapy, St. Petersburg State Academy of Veterinary Medicine, St. Petersburg, Russia
}

Article history

Received: 28-11-2016

Revised: 28-02-2017

Accepted: 09-03-2017

Email: wolfberg.guard@gmail.com

\section{Introduction}

Heart failure, despite its etiology, is coupled with significant disturbances of cardiomyocytes energy supply. Thank to Hermann and Dechred (1939) we keep in mind that heart failure is a sequence of myocytes energy starvation.

Myocardium is one of the most energy-depended structure, it demands about $6 \mathrm{~kg}$ of ATP a day. In order to sustain efficient energy supply it has advanced system producing enough ATP. First step is consuming of glucose and Free Fatty Acids (FFA) from blood, then glycolysis and beta-oxidation provide intermediates for

\begin{abstract}
Dilated Cardiomyopathy (DCM) and Chronical Degenerative Valvular Disease (CDVD) are very common in dogs nowadays. However, due to modern level in oncology we can meet chemotherapy-induced pathogenic mechanisms underlying Doxorubicin-induced Cardiomyopathy (DoxCM) and features differentiating it from CDVD and DCM. In this study we investigate several metabolic features of doxorubicin-induced cardiomyopathy, DCM-like phenotype cardiomyopathies and CDVD. We observed some stages of myocardium glucose metabolism in order to estimate life potential of end stage myocardium and respectively survival of patient. The study population consisted of 46 dogs of different age, sex and breed. These dogs were subgrouped due to diagnosis: Healthy dogs without heart failure, DCM, CDVD and DoxCM. Fresh myocardial biopsies taken immediately after euthanasia from left ventricular apex, freezed in liquid nitrogen for ELISA and biochemical study. Differences in metabolic profile found between of CDVD and healthy dogs were not significant. At the same time doxorubicin induced cardiomyopathy and DCM-like phenotype myocardial disease had similar changes. It should be mentioned, DCM patients had previously long history of treatment, but DoxCM group-not. Differences between DoxCM, DCM and CDVD were significant and can be explained by changes what suffering myocardium undergoes. Rapid or slow developing energy depletion leads to myocardiocytes death and heart failure. Doxorubicin-induced cardiomyopathy is far more dangerous due to rapidness of development in observed study. Myocardium, due to lack of time, had no adapting ability for acute energy depletion and massive cell death. This preliminary study shows changes induced by doxorubicin (Dox) in dogs. These findings mostly connected with mitochondrial disturbances, insulin resistance and energy depletion. In this study also shown several features connected with development of CDVD and differences from DCM.
\end{abstract}

Keywords: Myocardial Metabolism, Cardiac Histology, Cardiac Ultrastructure, Doxorubicin-Induced Cardiomyopathy
Tricarboxylic Acid Cycle (TCA). After that metabolites driven to mitochondrial oxidative chain, which phosphorylate ADP to ATP. In the end, Creatine Kinase (CK) system organize transport of macroergic phosphates from mitochondria to contractile sites. Phosphocreatine (PCr) is the most important substrate to support energy demands of myocardium (Ingwall and Weiss, 2004; Ingwall, 2002; 2009; Lopaschuk et al., 2002; Paolisso et al., 1994; Ventura-Clapier et al., 2004).

Oxidative phosphorylation-is the main source of macroergic phosphates. ATP synthesis is strongly connected with formation of Reactive Oxygen Species (ROS), what usually utilized by cell antioxidant system. 
Disturbances in mitochondrial oxidation leads to heart failure, due to decreased ATP production for myofibrils. Following changes are associated with structural damage of mitochondria, lost of its membrane potential, in the end we have decreased affinity to phosphorylation recipients (ADP, AMP, creatine) (Ingwall and Weiss, 2004; Ingwall, 2002; 2009; Lopaschuk et al., 2002; Ventura-Clapier et al., 2004).

Contractility suppress leads to activation of catecholamine supportive system. Activation of betaadrenergic receptors increases heart rate and ATP consumption, this situation ends with high amounts of ADP and negative inotropic effect (Liu et al., 2001; Neubauer, 2007).

There are lots of therapeutic agents with cardiotoxic effect. The significant amount of these drugs are used in oncology, due to its negative effect on neoplasia cells. Unfortunately, it is very difficult to organize target action of chemotherapy, so toxicity is mostly have effect on whole organism. Due to ROS-producing effect of chemotherapy agents, genetic apparatus of active dividing cells is damaged in both neoplasia cells and normally proliferating blood cells. Also high amounts of produced ROS are toxic to cells which highly depend on oxidative phosphorylation. Cardiomyocytes are one of them, it need to be mentioned, their vulnerability to chemotherapy drugs is also connected with very low cell antioxidant system activity (Takemura and Fujiwara, 2007; Yeh et al., 2004; Zucchi and Danesi, 2003).

As in human medicine, anthracyclines are most common chemotherapy drugs. Doxorubicin is leading agent due to its wide action specter. Unfortunately, chemotherapy always coupled by toxic side effects, which has significant influence on therapy quality and can a cause change of standard scheme of treatment (Aries et al., 2004; Lebrecht et al., 2005; Minotti et al., 2004; Takemura and Fujiwara, 2007; Yeh et al., 2004).

Negative effects of anthracyclines have influence on many organs and systems, significant alterations are usually connected with gastro-intestinal, hemopoetic and cardiovascular systems. Due to the fact that most oncology patients are geriatric, predispose to pay more attention to myocardium condition (Klimas, 2012; Takemura and Fujiwara, 2007).

Standard echocardiography and electrocardiography sometimes is not sufficient to find developing disease, due to inability to define state of individual cardiomyocytes, which could become sites of fibrosis and ectopic electro activity. Doxorubicin toxic effects are less common in dogs than in human, due to differences in chemotherapy scheme. In human medicine DoxCM often develop in several decades after treatment and in case of overcoming cumulative threshold of $450-500 \mathrm{mg} / \mathrm{m}^{2}$ (Swain et al., 2003). In veterinary medicine threshold dose is about $160-200 \mathrm{mg} \backslash \mathrm{m}^{2}$ (Mauldin et al., 1992).
In practice, we have two types of cardiotoxicity: Acute-connected with myocardial electroactivity disturbances and arrhythmias, which have to be medicated; and chronic toxicity-leading to cardiomyocytes death, fibrosis, systolic and diastolic dysfunction and metabolic disturbances in rest of myocardial cells (Swain et al., 2003; Takemura and Fujiwara, 2007; Von Hoff et al., 1979).

The leading pathogenesis mechanism of doxorubicin toxicity is still unknown, but already have been defined key pathological effects of anthracycline: Synthesis of reactive oxygen species (mitochondrial, No-connected, NAD(P)H-depended); DNA intercalation; complex bound doxorubicin-iron; alteration of $\mathrm{Ca}$ ions transport is sarcoplasmic reticulum; decreasing of macroergic phosphates synthesis; endothelin dysfunction; myocardial remodeling; oxidative stress (Bachur et al., 1977; Doroshow, 1983; Fogli et al., 2004; Gewirtz, 1999; Hasinoff, 1998; Kelvin and James, 1986; Myers et al., 1977; Nohl et al., 2003; Outomuro et al., 2007; Tokarska-Schlattner et al., 2006; Weinstein et al., 2000; Zhou et al., 2001b; 2001a; Zuppinger et al., 2007).

Compensatory mechanisms in developing cardiomyopathy include: Increasing in FFA consumption; adrenergic system activation; transcardial hemodynamics modulation; parenchymal and stromal remodeling.

From the first steps of heart failure, increased FFA consumption is coupled with catecholamine activation, until development of decompensation, which connected with metabolic turn to glycolysis (Ashrafian et al., 2007; Eisenhofer et al., 1996; Kostis and Sanders, 2005; Lopaschuk et al., 2010; Opie et al., 1979; Opie, 2006; Paolisso et al., 1991; Zucker, 2006).

FFA transport to cardiomyocytes is implemented by several ways: Passive diffusion and specific transport proteins. After diffusion of long-chain fatty acids in myocyte, they metabolized into acyl-Coenzyme A (acyl$\mathrm{CoA}$ ) and transported to specific protein on mitochondrial membrane for acyl-CoA synthase interaction. Active transport is supported by FATP1, FATP6, CD36, next step for this way obtained long-chain fatty acids is bounding with CoA and metabolizing in beta-oxidation. Some of these FFA are esterificated and stored as lipid drops in cytoplasm (Kampf et al., 2007; Luiken et al., 2002; 1999; 1997; van der Vusse et al., 2000).

Myocardial FFA metabolism is also influenced by secondary messenger AMP-Activated Protein Kinase (AMPK), which regulated by AMP $\backslash$ ATP ratio. There are several sites of this molecule action: Inhibition of malonyl-CoA, suppressing acyl-CoA synthase and decreasing of FFA storage; decreased malonyl-CoA prevent carnitine palmitoyltransferase inhibition and transport of FFA to mitochondria; stimulate expression of FATP and CD36 on outer cardiomyocyte membrane (Ardehali et al., 2012; 
Lionetti et al., 2011; Sack et al., 1996; Shibayama et al., 2015; Tokarska-Schlattner et al., 2005).

In addition, AMPK influences of glycolytic pathways of energy production: Increasing glucose transport due to activation of GLUT 1 and GLUT 4; inhibiting of glycogen synthesis by deactivation of glycogen-synthase (Anwan and Saggerson, 1993; Bonen et al., 1999; Fueger et al., 2007; Long et al., 2005; Luiken et al., 2003; Saha et al., 2000; Shearer et al., 2005; Steinberg and Kemp, 2009; Stoppani et al., 2002).

Family of glucose transporters in myocardium is mostly represented by GLUT 1, GLUT 4, GLUT 12. GLUT1 are insulin-independed and connected with base glucose supplementation. GLUT 12 is weakly expressed on cardiomyocyte outer membrane, but its role still unclear (Becker et al., 2001; Bell et al., 1993; Fischer et al., 1997; Stanley et al., 1997; Wheeler et al., 1994).

Nowadays role of GLUT4 is most significant in metabolic aspects of cardiomyopathies and local Insulin Resistance (IR) development. This type of transporter implements glucose supplementation to cytoplasm organelles, especially for T-tubules, due to group of associated $\mathrm{Ca}+2$ ion pumps which demand high amounts of ATP, got mostly from glycolysis (Fazakerley et al., 2009; Lauritzen et al., 2006; 2008; Wang et al., 1996).

Important step in understanding of metabolic part of heart failure is development of local IR. This state is based on persistent hyperinsulinemia. Adrenergic reaction coupled with heart failure leads to activation of glycomobilization system and Ins co-activation and, in parallel, catheholamine-induced lipomobilization (noradrenalin). Ins stimulate exocytosis of GLUT4 and CD36, which helps to get sufficient amounts of energy substrates for oxidative phosphorylation and glycolysis. But due to difference in Ins receptors we can get IR. This effect based on affinity of receptor to Ins, during chronic hyperinsulinemia receptors with low affinity suppress more active and worsen IR (Cook et al., 2010; Davila-Roman et al., 2002; De Meyts et al., 1976; 1973; Lionetti et al., 2007; Nikolaidis et al., 2004; Shanik et al., 2008; Taylor et al., 2001; Witteles et al., 2004).

In parallel, Ins-stimulated FFA consumption leads to their storage in cytoplasm, formation of lipid drops, increasing of acyl-CoA- FFA bounds concentration. These metabolites inhibit Pyruvate Dehydrogenase (PDH) activity and suppress glycolysis and exceed oxidative potential of mitochondria. Due to $\mathrm{PDH}$ inhibition glycolysis intermediate compounds are redirected to glycogen genesis, what brings accommodation of glycogen, inability to use it energy supplement, electroactivity disturbances because of storage disease. Also metabolites could act as a stimulators of hexosamine biosynthesis. Fructose-6phosphate is metabolized to uridinediphosphate-Nacetylglucosamine, what stimulates $\mathrm{O}$-associate glycolisation of contractile filaments, $\mathrm{Ca}$ ion pumps of sarcoplasmic reticulum. In the end it leads to apoptosis (Ashrafian et al., 2007; Clark et al., 2003; Hu et al., 2005; Kotoulas et al., 2004; Lopaschuk et al., 2010; Nikolaidis et al., 2001; Randle et al., 1963; Rechelt et al., 2013; Xu et al., 2010).

Creatine kinase complex, which implement distribution of phosphates between ATP and creatine, also damages in pathological myocardium. Decreased activity of this enzyme suppress PCr $\backslash$ ATP ratio, what leads to low ability to transport phosphates to ADP (Lopaschuk et al., 2010; Nahrendorf et al., 2005; Neubauer et al., 1997; Oleynikov et al., 2015; Randle et al., 1963; Wallis et al., 2005).

Lactate-Lactate Dehydrogenase (LDH)-pyruvate system helps to obtain additive amounts of pyruvate in order to retain energy substrates or to withdraw exceed amounts of pyruvate, metabolizing it to lactate, obtained due to inhibition of PDH by Randle cycle reactions. In heart failure, high adrenergic stimulation and compensatory mechanisms increase utilization of FFA what leads to inhibition of PDH and idle pyruvate metabolized by LDH to lactate and shuttles it in circulating blood. This process decreases general amounts of pyruvate and lactate in cytosol and rise in lactatelpyruvate ratio, negatively alternating energy supplementation in submembrane ion pumps (Des Rosiers et al., 2011; Lopaschuk et al., 2010; Oleynikov et al., 2015; Randle et al., 1963; Shibayama et al., 2015).

Additive component of energy deficit is electrolyte disturbances in cardiomyocyte metabolism. Under adrenergic stimulation there is active calcium transport and, in parallel, sodium retention in cardiomyocyte. High amount of $\mathrm{Na}$ suppress activity if $\mathrm{Ca}+2 \backslash \mathrm{Na}+$ pump and secondary cardiomyocyte has significant $\mathrm{Ca}+2$ ions in cytoplasm. Also high $\mathrm{Na}$ concentration leads to membrane depolarization disturbances and myocyte edema (Pieske et al., 2002).

On the first stage increased intracellular $\mathrm{Ca}+2$ flux stimulates $\mathrm{Ca}+2$-induced $\mathrm{Ca}+2$ release. This mechanism helps to increase contractile ability in stress situations. In case of chronic stimulation, there is energy depletion of tubular system $\mathrm{Ca}+2$ ionic pump, due to its high dependence to glycolytic ATP production. It could be explained by the fact of PDH complex inhibition, described earlier. Absence of effective $\mathrm{Ca}+2$ elimination leads to diastolic dysfunction and depolarization alteration (Avero et al., 1995; Dodd et al., 1993; Fabiato, 1983; Feher and Lipford, 1985; Jenden and Fairhurst, 1969; Lewartowski et al., 1990; Meissner and Henderson, 1987; Northover, 1991; Sutko and Airey, 1996).

In common, metabolic aspects of heart failure are described in many works and the common view on metabolic side of cardiomyopathy pathogenesis is already exist. However, differences between primary and secondary cardiomyopathies are not defined (Neubauer, 2007). 
Further we need to elucidate myocardial dysfunctions appeared after doxorubicin treatment. In common anthracyclines can cause myofilaments damage, suppression of their resynthesis; energy metabolism alteration, following depletion of PCr $\backslash$ ATP ratio, AMPK inhibition; damage of lipid structures of mitochondria by ROS; $\mathrm{Ca}+2$ storage in mitochondrial matrix, their petrification (Hrelia et al., 2002; Ingwall, 2004; Minotti et al., 2004; Olson and Mushlin, 1990; Weinstein et al., 2000; Zou et al., 2003).

Acute effects of Dox are based on rapid $\mathrm{Ca}+2$ flux from sarcoplasmic reticulum, activation of $\mathrm{Ca}+2-$ dependened $\mathrm{Ca}+2$ channels and inhibition of concurrent attachment of $\mathrm{Mg}+2$ ions to $\mathrm{Ca}+2$ receptors. Dox-induced increasing of $\mathrm{Ca}+2$ in cytoplasm leads to $\mathrm{Na}$ retention, suppression of Na\K pump, cardiomyocyte edema and high risk of arrhythmogenicelectroactivity. Sometimes, Dox-induced alteration can cause damage of sarcoplasmic reticulum (Doroshow, 1991; Holmberg and Williams, 1990; Minotti et al., 2004; Nagasaki and Fleischer, 1989; Pessah et al., 1992; Zucchi and Ronca-Testoni, 1997).

Chronic effects are connected with high concentration of intracellular $\mathrm{Ca}+2$ and inability of $\mathrm{Ca}+2$-ATP-ase reorganize flux of calcium. This aspect leads to decrease of $\mathrm{Ca}+2$ sensibility and diastolic dysfunction.

One of most toxic of Dox metabolites-doxorubicinol, can bound with intracellular $\mathrm{Fe}$ ions. In conditions of intracellular acidosis, doxorubicinol bounds to $\mathrm{Fe}+3$, taking it from ferritin and activates Fe influx in the cell. Dox $-\mathrm{Fe}+3$ complex metabolizes and form $\mathrm{Dox}-\mathrm{Fe}+2$ bound, what can oxidase lipids of organelles skeleton, proteins, DNA and form ROS. Due to significant lack of antioxidant system in cardiomyocytes, Dox-Fe+2 complexes can play key-role in Dox-induced apoptosis (Kwok and Richardson, 2003; 2004; Minotti, 1993; Minotti et al., 1999; 1995; Olson and Mushlin, 1990; Olson et al., 1988; Xu et al., 2005).

Dox interactions with mitochondria leads to cristae damage, membrane potential alteration, $\mathrm{Ca}$ ions storage in matrix, production of singlet oxygen. All these aspects are causing not only loss of mitochondria as functional unit, but activate them as apoptosis inductors (cytochrome C) (Minotti et al., 2004; Olson and Mushlin, 1990; Wang et al., 2001).

Dox metabolites alternates DNA and significantly damage tRNA and mRNA function. These changes cause depletion of synthetic activity of organelles and destabilization of energy producing (Minotti et al., 2004; Olson and Mushlin, 1990; Olson et al., 1988; $\mathrm{Xu}$ et al., 2005).

Retention of beta-oxidation intermediates inhibit normal flux of glycolysis. Glucose metabolites, stored in cytoplasm, can cause acidosis and pathological glycogen resynthesis (Minotti et al., 2004; Oleynikov et al., 2015).

Combination of all the components leads to local and general contractile dysfunction. Myocardial remodeling due to Dox-induced apoptosis can cause disturbances in electroactivity. Ion misbalance and developing remodeling characterize diastolic dysfunction.

In this study we are going to elucidate several differences in myocardial metabolism, histopathology in different cases of heart failure.

\section{Animals, Materials, Methods}

In this study, we studied 46 dogs of different sex, age and breed. All the dogs were patients of veterinary clinics "Pride" and "Champion", Saint Petersburg, Russia. Groups were formed due to primary diagnosis: (1) Control (animals without cardiology problems, $\mathrm{n}=$ 14); (2) CDVD (animals with chronic degenerative valvulardisease, $\mathrm{n}=16$ ); (3) DCM (patients with idiopathic dilated cardiomyopathy and dilated cardiomyopathy-phenotype changes, $n=6$ ); (4) DoxCM (dogs, treated with doxorubicin, $\mathrm{n}=10$ ).

The aim of this study is to elucidate some morphological and metabolic aspects of each nosologic units. Myocardium for research needs was obtained immediately after euthanasia on autopsy.

Studied animals were diagnosed by standard methods (clinical findings, blood analyzes, radiography, echocardiography, electrocardiography). Patients in control group, didn't have diagnosed myocardial diseases and were euthanized due to low life quality. In other groups euthanasia were admitted in cases of endstage and refractory heart failure and inability to sustain live functions or by owners will. Obtaining of samples were agreed with owners.

Materials were obtained right after death. Samples were taken from left ventricular apex. Material for histology fixed in neutral formalin and Bouin solution, for ultramicroscopic study-in glutaraldehyde, for biochemistry and ELISA-liquid nitrogen and stored in $-25^{\circ} \mathrm{C}$. All material were studied in two months, due to prevent significant loss in investigated substrates.

Histology samples were standardly prepared and stained with hematoxylin and eosin, metal hematoxylin, picrofuchsin and by Mallory. Ultramicroscopy studies were done in standard technic.

During biochemistry analyze we used standard kits for estimation of lactate concentration, activity of LDH and CK (Olvex Diagnosticum). In addition, we studied obtained samples to define concentrations of ATP and GLUT 4 protein by methods and reagents offered CloudClone Corp. (USA).

Statistical analysis was made by using of Statistic 7.0, for non-parametric cases, we used Mann-Whitney criteria.

\section{Results}

During routine examination (echo-and electrocardiography) in control group there was no 
evidences of myocardial dysfunction (data not presented). Data obtained from tissue biochemistry analyzes was taken as physiological. In addition, our results were compared with literature resources, what helped to define metabolism disturbances in different diseases.

Estimated concentrations of lactate and LDH in control group were taken as physiological level glycolytic myocardial tissue processes (Table 1).

LDH shows us activity and main path of carbohydrate metabolism. In control group, evaluated activity of this enzyme did not differ from commonly accepted data. We can assume that these measurements show transformation of unused pyruvate into lactate. Creatine kinase elucidate processes cooperation of creation, transport and concentration of macroergic phosphates and shows availability of energy supply. In this group, CK level characterizes unaltered energy storing. ATP concentrations are not very high in myocardium, but it compensates due to high activity of cytosolic enzymes. In the control group level of ATP was not different from observed data (Table 2).

Main glucose transporters in cardiomyocytes are GLUT 1 and GLUT4. Due to not very significant variability of GLUT 1 glucose transport in different circumstances and its role in basal support of glucose, in this study we investigated changes in GLUT4 proteins expression, in view of its flexibility regarding to tissue condition and insulin sensitivity. There is no conventional data of myocardium tissue concentration of GLUT4 protein, so we extrapolated it from skeletal muscle, where it expression was $142 \pm 39 \mathrm{ng} \backslash \mathrm{mg}$ of wet tissue (Kim et al., 2005). In our study, we admitted GLUT4 protein concentration in alike diapason (Table 2).

Then we studied these parameters in animals with Chronic Degenerative Valvular Disease (CDVD). During statistical analyze we didn't define significant difference between control group and CDVD. This situation can be explained as CDVD even in terminal stages doesn't significant alter myocardial metabolism and it mostly compensated (Table 1 and 2). Therefore, we can define that main pathogenetic role in CDVD is played by volume overload. Decrease of systolic function is based on dysfunction of Frank-Starling mechanism, which plays significant role in organization of excessive amount of intravascular liquid.

It is necessary to admit that CDVD is usually associated with cardiomyocytes hypertrophy. This reaction is aimed on decreasing of left ventricular wall stress. While decompensation progress, adaptive gene expression is taking place in myocardial energy metabolism and protein synthesis, transforming it to fetal type. The most common changes include: Increasing of beta-MCH production, which contractility is not as flexible as it is in normal conditions; activation of $\mathrm{CK}$ BB-homoisomer synthesis, which has more affinity to
ADP and is more effective in stabilizing of ATP concentration. One of the most significant parameter of fetal gene expression shifting is altering of GLUT1/GLUT4 ratio. This is based on 2 facts: (1) Altered insulin sensitivity leads to decrease of GLUT4 trafficking to membrane, but in this condition the whole amount of GLUT4 protein doesn't change (what is taking place in CDVD); (2) absolute decreasing of GLUT4 proteins and compensatory increase of insulininsensitive GLUT1expression, what characterizes local insulin resistance on next stages.

In the end we have to admit that myocardial metabolism in CDVD stay mostly unchanged, but it should be keep in mind that it is based on several compensatory mechanisms and leads to decreased lability of energetic metabolism and lowering tolerance to exercises.

Then we should evaluate changes presented in myocardial tissue under anthracycline antibiotics treatment (Table 1 and 2).

Estimated lactate tissue concentration was significantly lower in compare with all other groups (control $\mathrm{p} \leq 0.01$; CDVD $p \leq 0.01$; DCM $p \leq 0.05$ ). This situation is based on enzymatic disturbances under doxorubicin toxicity. Tissue lactate concentration characterize intensity of glycolysis substrate supplementation and wastes utilization. In this case, we find low activity of glucose incoming, due to decreased GLUT4 protein appearance (control $\mathrm{p} \leq 0.01$; CDVD $p \leq 0.01 ; D C M p \geq 0.05$ ). Insufficiency of glucose supplementation leads to decreasing of intermedia metabolites production, mostly glucose-6-phosphate, which elucidate not only carbohydrate consuming, but also hexokinase activity, one of the weakest part of glycolysis chain.

In many available works were admitted that the main energy substrates during cardiomyopathy and heart failure progressing are shifting from free fatty acids to glucose. Hexokinase-is one of the threshold enzymes, taking part in carbohydrate metabolism regulation, during energy starvation hexokinase mRNA is significantly increase, bit its activity standstill, which is crucial limiting factor in glycolysis energy supply. Decreased glucose metabolism leads to lowering of pyruvate producing. This insufficiency has negative influence on TCA cycle, because even in normal conditions TCA intermedia metabolites (succinate, fumarate) leaks the cell, but in pathological situation and shortage of pyruvate makes this leakage irreversible and significant. Low concentrations of pyruvate leads to decreasing of lactate production. Therefore, we can say that low activity of $\mathrm{LDH}$ in case of DoxCM (control $\mathrm{p} \leq 0.01 ; \operatorname{CDVD} \mathrm{p} \leq 0.01 ; \operatorname{DCM} \mathrm{p} \geq 0.05$ ) is based on 2 facts: (1) Shortage in substrate, due to glycolysis suppression; (2) altering effect of doxorubicin on NADdepended dehydrogenase systems, this explanation also suitable for inability of pyruvate restoration by lactate and NAD metabolism. 
Table 1. Myocardial biochemistry parameters

\begin{tabular}{llll}
\hline Group & Lact, $\mu \mathrm{mol} / \mathrm{g}$ & $\mathrm{LDH}, \mathrm{U} / \mathrm{g}$ & $\mathrm{CK}, \mathrm{U} \backslash \mathrm{mg}$ \\
\hline Control $(\mathrm{n}=14)$ & $13,2 \pm 8,32$ & $251,31 \pm 55,94$ & $3,34 \pm 1,01$ \\
CDVD $(\mathrm{n}=16)$ & $13,44 \pm 4,22$ & $264,58 \pm 47,42$ & $3,93 \pm 0,55^{* *}$ \\
DCM $(\mathrm{n}=6)$ & $10,05 \pm 5,34++$ & $94,87 \pm 42,74^{*+}$ & $1,36 \pm 0,24^{*+}$ \\
DoxCM $(\mathrm{n}=10)$ & $8,84 \pm 4,7++$ & $54,76 \pm 20,65^{*+}$ & $2,1 \pm 0,54^{*+}, "$ \\
\hline
\end{tabular}

$*-p \leq 0,01$ to control

$* *-\mathrm{p} \leq 0,05$ to control

$+-p \leq 0,01$ to CDVD

$++-p \leq 0,05$ to CDVD

" $-\mathrm{p} \leq 0,01$ to DCM

"" $-\mathrm{p} \leq 0,05$ to DCM

Table 2. ATP and GLUT4 tissue concentraions

\begin{tabular}{|c|c|c|}
\hline Group & ATP, ng/g & GLUT 4, $\mathrm{ng} \mathrm{mg}^{-1}$ \\
\hline Control $(n=14)$ & $3,37 \pm 0,67$ & $138,68 \pm 88,81$ \\
\hline $\operatorname{CDVD}(\mathrm{n}=16)$ & $3,01 \pm 0,9$ & $106,59 \pm 64,97$ \\
\hline $\operatorname{DCM}(\mathrm{n}=6)$ & $0,59 \pm 0,59 *+$ & $51,69 \pm 20,76^{*+}$ \\
\hline $\operatorname{DoxCM}(n=10)$ & $0,27 \pm 0,1 *+$ & $45,21 \pm 11,0 *+$ \\
\hline \multicolumn{3}{|c|}{$\begin{array}{l}\text { * }-\mathrm{p} \leq 0,01 \text { to control } \\
* *-p \leq 0,05 \text { to control } \\
+-p \leq 0,01 \text { to CDVD } \\
++-p \leq 0,05 \text { to CDVD } \\
\text { "- }-\mathrm{p} \leq 0,01 \text { to DCM } \\
\text { "" }-\mathrm{p} \leq 0,05 \text { to DCM }\end{array}$} \\
\hline
\end{tabular}

It is known, there is an additive system, taking part in lactate supplementation for necessary pyruvate restorationmonocarboxylate transporters. However, in case of intracellular $\mathrm{pH}$ decreasing this structure is unable to transport available lactate, but let ketone bodies into cell.

It should be mentioned, there is one more imitating pyruvate utilization factor. Heart failure is usually associated with adrenergic stimulation for stroke volume compensation. Emission of catecholamine contribute to lipomobilization and increasing of serum free fatty acids concentration, which, due to their lipophilic nature, freely enter cardiomyocyte. Free fatty acid metabolites lead to PDH complex inhibition and abolish compensatory effects of glycolysis. In case of DoxCM it is complicated by the fact that Dox itself alters mitochondrial metabolism and leads to suppression of several transport systems, including carnitine palmitoiltransferase and NAD-depended enzymes. This blocks usage of absorbed free fatty acids in lipid betaoxidation and ATP resynthesis. So, fatty acids storage in cytosol as triglycerides. Energy demand and blockage of fatty acids utilization leads to ATP-wasting cycles of mobilization-stabilization of cytosolic triglycerides, ceramides and products of lipid peroxidation storage.

Mitochondrial apparatus and energy metabolism alteration leads to $\mathrm{CK}$ system dysfunction. Though CK changed to BB-homoisomer Dox still inactivates it and, in parallel, leads to cell free creatine leakage due to decreased its phosphorylation. Doxorubicin coupled with mitochondrial membrane cardiolipines competitive blocks mitochondrial CK adhesion, alters connection with cytochrome apparatus. This leads to significant uncoupling of macroergic bounds form mitochondria to creatine transport. This reaction blocks creatine phosphorylation near mitochondria and then lowering of energy transport to contractile components due to suppressing cytosolic and mitochondrial CK.

Further, low GLUT4 protein concentration and CK alteration are leading to inhibition of PDH and its connection with hexokinase, triglyceride storage and local insulin resistance appearance.

Insufficiency of glycolysis substrates stimulates GLUT4 exocytosis and trafficking from intracellular storage places, but high concentrations of cytosolic triglycerides aborts GLUT 4 vesicles transport and membrane exocytosis. In addition, alternates signal pathways of insulin receptors, associated with straight PIkinase inhibition, which controls GLUT4 mobilization.

Stress is associated with catecholamine emission, which stimulates hyperinsulinemia and hyperglycemia. Heart failure-is chronic stress, so it has own featureshyperglycemia is a rare fact, but hyperinsulinemia and tissue insulin resistance (in patients of 3 stage by NYHA classification) is mostly presented. This situation is accelerated by anthracycline. Doxorubicin stimulates corticosteroids secretion, decreases insulin receptors sensitivity, blocks AMPK phosphorylation and inhibit GLUT 4 trafficking to plasmolemma.

It is known, there are two forms of insulin receptorsA\&B. A form-is characterized high affinity to insulin and in normal conditions represent $95 \%$ of all insulin receptors in brain and heart tissues. B form- is less affine to the hormone and often follow pathological situations, associated with long-term hyperinsulinemia.

The significant influence insulin resistance has on contractile components of myocardium, due to high amounts of GLUT4 in T-tubules. GLUT4 activity depression and decrease of their exposition in tubules leads to suppression of glucose supply and blocking of ATP synthesis for tubular ionic pumps.

Combination of presented alterations lead to decrease of ATP synthesis, which is markedly represented in group of DoxCM (control $\mathrm{p} \leq 0.01$; CDVD $\mathrm{p} \leq 0.01$; DCM $\mathrm{p} \geq 0.05$ ).

Doxorubicin effects have influence on many vital functions of cardiomyocytes: 
- Dox oxidizes components of mitochondrial DNA, alternates mitochondria and their enzymes resynthesis

- Affecting on cellular iron, creates ROS, hydroxide groups, which alone or in association with $\mathrm{NO}$ intercalate DNA, competitively couple with cofactors, necessary for electron transport chain

- Primarily stimulates release of $\mathrm{Ca}+2$ ions from SR (acute faze, high risk of arrhythmias ), then suppress activity of $\mathrm{Ca}+2-\mathrm{ATP}$-ase, which leads to storage of $\mathrm{Ca}+2$ ions in cytosole and contracture of sarcomere

- Stimulates entrance and storage of $\mathrm{Ca}+2$ in mitochondria, alternating its membrane potential and petrification

- In different ways alternates macroergic phosphates synthesis, what is highly notable in enzyme-ionic pump complex, which is strongly connected with glycolysis

- Leads to ionic pumps dysfunction, associated with myocardial edema, membrane potential alteration

- Stimulates insulin resistance appearance

Further we evaluated and estimated metabolic parameters in cases of DCM (Table 1 and 2). Received data shows significant changes in myocardial condition. We should note some aspects. Glycolysis in this situation is characterized with lower alternation, than in DoxCM group, this may be connected with less active damage of energy supplementation enzyme systems in absence of doxorubicin. GLUT4 protein concentration is much lower in compere with control and CDVD groups, but lactate, LDH, ATP measurements elucidate how cardiomyocytes trying to normalize their metabolism by glycolysis. It should be noted, DCM also characterized with insulin resistance, but it is a long-term process.

\section{Discussion and Conclusion}

Myocardium metabolism in control group characterized with normal glycolytic activity and more active oxidative phosphorylation, reflected in physiological ATP concentration. GLUT 4 protein concentration was alike to muscle concentration, so we extrapolated this data on myocardium and applied it as physiological state. We didn't define differences between membrane and cytosolic forms of GLUT 4 and their trafficking under insulin stimulation. However, due to normal ATP concentration and systolic function we admitted that GLUT 4 function was not altered.

In CDVD group, we defined statistically nonsignificant changes in studied parameters. This data characterized compensated state of myocardial energy metabolism. Nevertheless, referencing to available data, we can say that animals with decompensated state (retroand anterograde heart failure), have 3 and higher stage in NYHA grades of failure, had myocardial IR (Friehs et al., 1990; Kolwitcz and Tian, 2011; Paternostro et al., 1999;
Razeghi et al., 2001; Schlattner et al., 1998; Tokarska-Schlattner et al., 2005; Wallimann et al., 1999). Despite normal concentration of GLUT4 proteins, their activity and Ins response had been suppressed, what leaded to low flexibility of myocardial metabolism during exercises (exercise intolerance, dyspnea, muscle weakness).

Data obtained from DCM group characterized significant metabolic alterations. All investigated parameters were suppressed, except lactate concentration. This feature have to be connected with chronic development of this pathology and following energy substrate changing to glucose. Despite decreased GLUT 4 concentrations, level of produced lactate reflects ability to utilize carbohydrates for energy supplementation. Of course, it was not enough and energy producing is severely decreased, first of all due to mitochondria dysfunction. In comparison to our data there is an experimental work, what showed increasing in DCM myocardial GLUT 4 proteins concentration. We can't refute this possible compensatory reaction, but this findings could be connected with absence of significant decompensation due to little period of development. This means, in order to have stable genetic reaction and activation of fetal myocardium genetic pattern, activation of glycolytic path of energy supplementation and its exhaust myocardium needs some time.

In our study, DCM group didn't have marked compensatory AMPK-stimulated reactions, due to IR and cycling energy-wasting reactions of triglyceride mobilization-storage in cell (Minotti et al., 2004; Shibayama et al., 2015; van der Vusse et al., 2000). Low activity of CK, ATP concentration characterized myocardial energy starvation and following contractile dysfunction (Garcia et al., 1994; Hawkins, 1971; Johannsson et al., 1997; Osorio et al., 2002; Wilson et al., 1987). Combination of damaging factors leaded to massive cardiomyocytes apoptosis, adipose and connective tissue proliferation and fibrosis.

In DoxCM group, we observed significant decrease of all studied metabolic parameters. Obtained data characterized severe Dox-induced toxicity on cardiomyocytes in investigated cases.

In contrast to DCM, Dox toxicity develops in comparatively short period, so interpretation of received data is quite difficult. In case of DoxCM IR plays more important role on initial stages of heart failure, when utilization of FFA is increased, because of Dox-induced alteration of oxygen-depended components of myocardial energy metabolism. Increased consumption of FFA, mitochondrial dysfunction lead to cell triglyceride storage, suppression of PDH complex, disorders in GLUT 4 transport and membrane bound, Ins insensibility (de Lima Junior et al., 2016; Randle et al., 1963; Scwenk and Luiken, 2008). PDH block leads to inability to use carbohydrates as energy substrate and compensate 
metabolic insufficiency (Tokarska-Schlattner et al., 2005; Wallimann et al., 1999). In addition, Dox toxicity causes impaired transport and enzyme proteins synthesis, negatively affect function of already presented proteins. This leads to degeneration of cell contractile components, vesicles formation and activation of phagolysosomes. In addition, Dox-induce: Retention of $\mathrm{Na}$ and stimulates cardiomyocyte edema: Change in cellstored Fe activity and ROS generation; $\mathrm{Ca}+2$ storage in sarcoplasm and following contracture, $\mathrm{Ca}+2$ insensibility of myofilaments; increased retention of calcium ions in mitochondria leading to membrane potential disturbances and petrification.

\section{Acknowledgment}

Special thanks to Toropova J.V., Zelinskaya I.V. for providing an opportunity to analyze studied substrates; Korolkova E.D. for help, advices and consultations.

\section{Ethics}

This article is original and contains unpublished material. The corresponding author confirms that all of the other authors have read and approved the manuscript and no ethical issues involved.

\section{References}

Anwan, M.M. and D.E. Saggerson, 1993. Malonyl-CoA metabolism in cardiac myocytes and its relevance to the control of fatty acid oxidation. Biochem. J., 295: 61-65. DOI: 10.1042/bj2950061

Ardehali, H., H.N. Sabbah, M.A. Burke, S. Sarma and P.P. Liu et al., 2012. Targeting myocardial substrate metabolism in heart failure: Potential for new therapies. Eur. J. Heart Fail., 14: 120-129. DOI: $10.1093 /$ eurjhf/hfr 173

Aries, A., P. Paradis, C. Lefebvre, R.J. Schwartz and M. Nemer, 2004. Essential role of GATA-4 in cell survival and drug-induced cardiotoxicity. Proc. Natl. Acad. Sci. USA, 101: 6975-6980.

DOI: $10.1073 /$ pnas.0401833101

Ashrafian, H., M.P. Frennaux and L.H. Opie, 2007. Metabolic mechanisms in heart failure. Circ, 116: 434-448.

DOI: 10.1161/CIRCULATIONAHA.107.702795

Avero, T.G., A.C. Zable and J.J. Abramson, 1995. hydrogen peroxide stimulates the $\mathrm{Ca}^{2+}$ release channel from skeletal muscle sarcoplasmic reticulum $^{(*)}$. J. Biol. Chem., 270: 25557-25563. DOI: $10.1074 /$ jbc.270.43.25557

Bachur, N.R., S.L. Gordon and M.V. Gee, 1977. Anthracycline antibiotic augmentation of microsomal electron transport and free radical formation. Mol. Pharmacol., 13: 901-910. PMID: 19695
Becker, C., L. Sevilla, E. Tomas, M. Palacin and A. Zorzano et al., 2001. The endosomal compartment is an insulin-sensitive recruitment site for GLUT4 and GLUT1 glucose transporters in cardiac myocytes. Endocrinology, 142: 5267-5276.

DOI: 10.1210 /endo.142.12.8555

Bell, G.L., C.F. Burant, J. Takeda and G.W. Gould, 1993. Structure and function of mammalian facilitative sugar transporters. J. Biocl. Chem., 268: 19161-19164. PMID: 8366068

Bonen, A., D.J. Dyck, A. Ibrahimi and N.A. Abumurad, 1999. Muscle contractile activity increases fatty acid metabolism and transport and FAT/CD36. Am. J. Physiol. Endocrinol. Metab., 276: E642-E649. PMID: 10198299

Clark, R.J., P.M. McDonough, E. Swanson, S.U. Trost and M. Suzuki et al., 2003. Diabetes and the accompanying hyperglycemia impairs cardiomyocyte calcium cycling through increased nuclear $O$-GlcNAcylation. J. Biol. Chem., 278: 44230-44237. DOI: 10.1074/jbc.M303810200

Cook, S.A., A. Varela-Carver, M. Mongillo, C. Kleinert and M.T. Khan et al., 2010. Abnormal myocardial insulin signalling in type 2 diabetes and leftventricular dysfunction. Eur. Heart J., 31: 100-111. DOI: 10.1093/eurheartj/ehp396

Davila-Roman, V.G., G. Vedala, P. Herrero, L. de las Fuentes and J.G. Rogers et al., 2002. Altered myocardial fatty acid and glucose metabolism in idiopathic dilated cardiomyopathy. J. Am. College Cardiol., 40: 271-277. PMID: 12106931

de Lima Junior, E.A., A.S. Yamashita, G.D. Pimentel, L.G. De Sousa and R.V. Santos et al., 2016. Doxorubicin caused severe hyperglycaemia and insulin resistance, mediated by inhibition in AMPk signalling in skeletal muscle. J. Cachexia, Sarcopenia Muscle, 7: 615-625. PMID: 27239415

De Meyts, P., A.R. Bianco and J. Roth, 1976. Site-site interactions among insulin receptors: Characterization of the negative cooperativity. J. Biol. Chem., 251: 1877-1888. PMID: 5434

De Meyts, P., J. Roth, D.M. Neville Jr, J.R. Gavin and M.A. Lesniak, 1973. Insulin interactions with its receptors: Experimental evidence for negative cooperativity. Biochem. Biophys. Res. Commun., 55: 154-161. DOI: 10.1016/S0006-291X(73)80072-5

Des Rosiers, C., F. Labarthe, S.G. Lloyd and J.C. Chatham, 2011. Cardiac anaplerosis in health and disease: Food for thought. Cardiovasc. Res., 90: 210-219. DOI: $10.1093 / \mathrm{cvr} / \mathrm{cvr} 055$

Dodd, D.A., J.B. Atkinson, R.D. Olson, S. Buck and B.J. Cusack et al., 1993. Doxorubicin cardiomyopathy is associated with a decrease in calcium release channel of the sarcoplasmic reticulum in a chronic rabbit model. J. Clin. Invest., 91: 1697-1705. DOI: $10.1172 /$ JCI116379 
Doroshow, J.H., 1983. Effect of anthracycline antibiotics on oxygen radical formation in rat heart. Cancer Res., 43: 460-472. PMID: 6293697

Doroshow, J.H., 1991. Doxorubicin-induced cardiac toxicity. N. Engl. J. Med., 324: 843-845. DOI: $10.1056 /$ NEJM199103213241210

Eisenhofer, G., P. Friberg, B. Rundqvist, A.A. Quyyumi and G. Lambert et al., 1996. Cardiac sympathetic nerve function in congestive heart failure. Circulation, 93: 1667-1676. DOI: 10.1161/01.CIR.93.9.1667

Fabiato, A., 1983. Calcium-induced release of calcium from the cardiac sarcoplasmic reticulum. Am. J. Physiol., 245: C1-C14. PMID: 6346892

Fazakerley, D.J., S.P. Lawrence, V.A. Lizunov, S.W. Cushman and G.D. Holman, 2009. A common trafficking route for GLUT4 in cardiomyocytes in response to insulin, contraction and energy-status signalling. J. Cell Sci., 122: 727-734.

DOI: $10.1242 /$ jcs.041178

Feher, J.J. and G.B. Lipford, 1985. Mechanism of action of ryanodine on cardiac sarcoplasmic reticulum. Biochim. Biophys. Acta, 813: 77-86. DOI: $10.1016 / 0005-2736(85) 90347-5$

Fischer, Y., J. Thomas, L. Sevilla, P. Munoz and C. Becker et al., 1997. Insulin-induced recruitment of glucose transporter 4 (GLUT4) and GLUT1 in isolated rat cardiac myocytes: Evidence of the existence of different intracellular GLUT4 vesicle populations. J. Biol. Chem., 272: 7085-7092. DOI: $10.1074 /$ jbc.272.11.7085

Fogli, S., P. Nieri and M.C. Breschi, 2004. The role of nitric oxide in anthracycline toxicity and prospects for pharmacologic prevention of cardiac damage. FASEB J., 18: 664-675. PMID: 15054088

Friehs, I., A.M. Moran, C. Stamm, S.D. Colan and K. Takeuchi et al., 1990. Impaired glucose transporter activity in pressure-overload hypertrophy is an early indicator of progression to failure. Circ, 100: 187-193. PMID: 10567302

Fueger, P.T., R.S. Lee-Young, J. Shearer, D.P. Bracy and S. Heikkinen et al., 2007. Phosphorylation barriers to skeletal and cardiac muscle glucose uptakes in high-fat fed mice: Studies in mice with a $50 \%$ reduction of hexokinase II. Diabetes, 56: 2476-2484. DOI: $10.2337 / \mathrm{db} 07-0532$

Garcia, C.K., J.L. Goldstein, R.K. Pathak, R.G. Anderson and M.S. Brown, 1994. Molecular characterization of a membrane transporter for lactate, pyruvate and other monocarboxylates: Implications for the Cori cycle. Cell, 76: 865-873. DOI: 10.1016/0092-8674(94)90361-1

Gewirtz, D.A., 1999. A critical evaluation of the mechanisms of action proposed for the antitumor effects of the anthracycline antibiotics adriamycin and daunorubicin. Biochem. Pharmacol., 57: 727-741. DOI: $10.1016 / \mathrm{S} 0006-2952(98) 00307-4$
Hasinoff, B.B., 1998. Chemistry of dexrazoxane and analogues. Semin. Oncol., 25: 3-9. PMID: 9768817

Hawkins, R.A., 1971. Uptake of ketone bodies by rat brain in vivo. Biochem. J., 121: 17-17. PMID: 5116532

Hermann, G. and G.M. Dechred, 1939. The chemical nature of heart failure. Ann. Inter. Med., 12: 1233-1244.

Holmberg, S.R.M. and A.J. Williams, 1990. Patterns of interaction between anthraquinone drugs and the calcium-release channel from cardiac sarcoplasmic reticulum. Circ. Res., 67: 272-283. PMID: 2165441

Hrelia, S., D. Fiorentini, T. Maraldi, C. Angeloni and A. Bordoni et al., 2002. Doxorubicin induces early lipid peroxidation associated with changes in glucose transport in cultured cardiomyocytes. Biochim. Biophys. Acta, 1567: 150-156. PMID: 12488048

Hu, Y., D. Belke, J. Suarez, E. Swanson and R. Clark et al., 2005. Adenovirus-mediated overexpression of O-GlcNAcase improves contractile function in the diabetic heart. Circ Res., 96: 1006-1013. PMID: 15817886

Ingwall, J.S. and R.G. Weiss, 2004. Is the failing heart energy starved? On using chemical energy to support cardiac function. Circ. Res., 95: 135-145. PMID: 15271865

Ingwall, J.S., 2002. ATP and the Heart. 1st Edn., Springer Science \& Business Media, Boston, ISBN-10: 1402070934, pp: 244.

Ingwall, J.S., 2009. Energy metabolism in heart failure and remodelling. Cardiovasc Res., 81: 412-419. PMID: 18987051

Ingwall, J.S., 2004. Transgenesis and cardiac energetics: New insights into cardiac metabolism. J. Mol. Cell. Cardiol., 37: 613-623. PMID: 15350834

Klimas, J., 2012. Drug-Induced Cardiomyopathies. INTECH Open Access Publisher, ISBN-10: 9533078340.

Jenden, D.J. and A.S. Fairhurst, 1969. The pharmacology of ryanodine. Pharmacol. Rev., 21: 1-25. PMID: 4887724

Johannsson, E., E.A. Nagelhus, K.J. McCullagh, O.M. Sejersted and T.W. Blackstad et al., 1997. Cellular and subcellular expression of the monocarboxylate transporter MCT1 in rat heart: A high-resolution immunogold analysis. Circ. Res., 80: 400-407. DOI: 10.1161/01.res.0000435856.47954.71

Kampf, J.P., D. Parmley and A.M. Kleinfeld, 2007. Free fatty acid transport across adipocytes is mediated by an unknown membrane protein pump. Am. J. Physiol. Endocrinol. Metab., 293: E1207-E1214. PMID: 17711989

Kelvin, J.D. and H.D. James, 1986. Redox cycling of anthracyclines by cardiac mitochondria. I. Anthracycline radical formation by NADH dehydrogenase. J. Biocl. Chem., 261: 3060-3067. PMID: 3456345 
Kolwitcz, S.C. and R. Tian, 2011. Glucose metabolism and cardiac hypertrophy. Cardiovascular Res., 90: 194-201. DOI: $10.1093 / \mathrm{cvr} / \mathrm{cvr} 071$

Kostis, J.B. and M. Sanders, 2005. The association of heart failure with insulin resistance and the development of type 2 diabetes. Am. J. Hypertens., 18: 731-737. PMID: 15882558

Kotoulas, O.B., S.A. Kalamidas and D.J. Kondomerkos, 2004. Glycogen autophagy. Microsc. Res. Tech., 64: $10-20$.

Kwok, J.C. and D.R. Richardson, 2003. Anthracyclines induce accumulation of iron in ferritin in myocardial and neoplastic cells: Inhibition of the ferritin iron mobilization pathway. Mol. Pharmacol., 63: 849-861. PMID: 12644586

Kwok, J.C. and D.R. Richardson, 2004. Examination of the mechanism(s) involved in doxorubicin-mediated iron accumulation in ferritin: Studies using metabolic inhibitors, protein synthesis inhibitors and lysosomotropic agents. Mol. Pharmacol., 65: 181-195. PMID: 14722250

Lauritzen, H., T. Ploug, C. Prats, J.M. Tavaré and H. Galbo, 2006. Imaging of insulin signaling in skeletal muscle of living mice shows major role of $\mathrm{T}$ tubules. Diabetes, 55: 1300-1306. PMID: 16644686

Lauritzen, H., T. Ploug, J. Brandauer, L.J. Goodyear and T. Ploug, 2008. Large GLUT4 vesicles are stationary while locally and reversibly depleted during transient insulin stimulation of skeletal muscle of living mice: Imaging analysis of GLUT4enhanced green fluorescent protein vesicle dynamics. Diabetes, 57: 315-324. PMID: 17977960

Lebrecht, D., A. Kokkori, U.P. Ketelsen, B. Setzer and U.A. Walker, 2005. Tissue-specific mtDNA lesions and radical-associated mitochondrial dysfunction in human hearts exposed to doxorubicin. J. Pathol., 207: 436-444. PMID: 16278810

Lewartowski, B., R.G. Hansford, G.A. Langer and E.G. Lakatta, 1990. Contraction and sarcoplasmic reticulum $\mathrm{Ca}^{2+}$ content in single myocytes of guinea pig heart: Effect of ryanodine. Am. J. Physiol., 259: H1222-H1229. PMID: 2221128

Lionetti, V., L. Guiducci, G.D. Aquaro, C. Simi and D. De Marchi et al., 2007. Mismatch between uniform increase in cardiac glucose uptake and regional contractile dysfunction in pacing-induced heart failure. Am. J. Physiol. Heart, 293: 2747-2756. PMID: 17704291

Lionetti, V., W.C. Stanley and F.A. Recchia, 2011. Modulating fatty acid oxidation in heart failure. Cardiovasc Res., 90: 202-209.

Liu, J., C. Wang, Y. Murakami, G. Gong and Y. Ishibashi et al., 2001. Mitochondrial ATPase and high-energy phosphates in failing hearts. Am. J. Physiol. Heart Circ. Physiol., 281: H1319-H1326. PMID: 11514303
Long, Y.C., B.R. Barnes, M. Mahlapuu, T.L. Steiler and S. Martinsson et al., 2005. Role of AMP-activated protein kinase in the coordinated expression of genes controlling glucose and lipid metabolism in mouse white skeletal muscle. Diabetologia, 48: 2354-2364. DOI: 10.1007/s00125-005-1962-5

Lopaschuk, G.D., I.M. Rebeyka and M.F. Allard, 2002. Metabolic modulation: A means to mend a broken heart. Circulation, 105: 140-142. PMID: 11790689

Lopaschuk, G.D., J.R. Ussher, C.D. Folmes, J.S. Jaswal and W.C. Stanley, 2010. Myocardial fatty acid metabolism in health and disease. Physiol. Rev., 90: 207-258. DOI: 10.1152/physrev.00015.2009

Luiken, J.J., S.L.M. Coort, J. Willems, W.A. Coumans and A. Bonen et al., 2003. Contraction-Induced fatty acid translocase/CD36 translocation in rat cardiac myocytes is mediated through ampactivated protein kinase signaling. Diabetes, 52: 1627-1634. DOI: 10.2337/diabetes.52.7.1627

Luiken, J.J., D.P. Koonen, J. Willems, A. Zorzano and C. Becker et al., 2002. Insulin stimulates longchain fatty acid utilization by rat cardiac myocytes through cellular redistribution of FAT/CD36. Diabetes, 51: 3113-3119.

DOI: $10.2337 /$ diabetes.51.10.3113

Luiken, J.J., F.G. Schaap, F.A. van Nieuwenhoven, G.J. van der Vusse and A. Bonen et al., 1999. Cellular fatty acid transport in heart and skeletal muscle as facilitated by proteins. Lipids, 34: S169-S175. DOI: $10.1007 /$ BF02562278

Luiken, J.J., F.A. van Nieuwenhoven, G. America, G.J. van der Vusse and J.F. Glatz, 1997. Uptake and metabolism of palmitate by isolated cardiac myocytes from adult rats: Involvement of sarcolemmal proteins. J. Lipid. Res., 38: 745-758. PMID: 9144089

Mauldin, G.E., P.R. Fox, A.K. Patnaik, B.R. Bond and S.C. Mooney et al., 1992. Doxorubicin-lnduced cardiotoxicosis clinical features in 32 dogs. J. Vet. Intern. Med., 6: 82-88.

DOI: $10.1111 / \mathrm{j} .1939-1676.1992 . t b 03156 . x$

Meissner, G. and J.S. Henderson, 1987. Rapid calcium release from cardiac sarcoplasmic reticulum vesicles is dependent on $\mathrm{Ca}^{2+}$ and is modulated by $\mathrm{Mg}^{2+}$, adenine nucleotide and calmodulin. J. Biol. Chem., 262: 3065-3073. PMID: 2434495

Minotti, G., 1993. Sources and role of iron in lipid peroxidation. Chem. Res. Toxicol., 6: 134-146. DOI: $10.1021 / \mathrm{tx} 00032 \mathrm{a} 001$

Minotti, G., G. Cairo and E. Monti, 1999. Role of iron in anthracycline cardiotoxicity: New tunes for an old song? FASEB J., 13: 199-212. PMID: 9973309 
Minotti, G., A.F. Cavaliere, A. Mordente, M. Rossi and R. Schiavello et al., 1995. Secondary alcohol metabolites mediate iron delocalization in cytosolic fractions of myocardial biopsies exposed to anticancer anthracyclines. Novel linkage between anthracycline metabolism and iron-induced cardiotoxicity. J. Clin. Investig., 95: 1595-1605. PMID: 7706466

Minotti, G., P. Menna, E. Salvatorelli, G. Cairo and L. Gianni, 2004. Anthracyclines: Molecular advances and pharmacologic developments in antitumor activity and cardiotoxicity. Pharmacol. Rev., 56: 185-229. DOI: 10.1124/pr.56.2.6

Myers, C.E., W.P. McGuire, R.H. Liss, I. Ifrim and K. Grotzinger et al., 1977. Adriamycin: The role of lipid peroxidation in cardiac toxicity and tumor response. Science, 197: 165-167. DOI: $10.1126 /$ science. 877547

Nagasaki, K. and S. Fleischer, 1989. Modulation of the calcium release channel of sarcoplasmic reticulum by adriamycin and other drugs. Cell Calcium, 10: 63-70. DOI: 10.1016/0143-4160(89)90045-6

Nahrendorf, M., M. Spindler, K. Hu, L. Bauer and O. Ritter et al., 2005. Creatine kinase knockout mice show left ventricular hypertrophy and dilatation, but unaltered remodeling post-myocardial infarction. Cardiovasc Res., 65: 419-427.

DOI: 10.1016/j.cardiores.2004.10.006

Neubauer, S., M. Horn, M. Cramer, K. Harre and J.B. Newell et al., 1997. Myocardial phosphocreatine-to ATP ratio is a predictor of mortality in patients with dilated cardiomyopathy. Circulation, 96: 2190-2196. DOI: 10.1161/01.CIR.96.7.2190

Neubauer, S., 2007. The failing heart - An engine out of fuel. N Engl. J. Med., 356: 1140-1151. DOI: $10.1056 /$ NEJMra063052

Nikolaidis, L.A., T. Hentosz and R. Huerbim, 2001. Mechanisms whereby rapid RV pacing causes LV dysfunction: Perfusion-contraction matching and NO. Am. J. Physiol. Heart. Circ. Physiol., 281: 2270-2281. PMID: 11709392

Nikolaidis, L.A., A. Sturzu, C. Stolarski, D. Elahi and Y.T. Shen et al., The development of myocardial insulin resistance in conscious dogs with advanced dilated cardiomyopathy. Cardivascular Res., 61: 297-306. DOI: 10.1016/j.cardiores.2003.11.027

Nohl, H., L. Gille, A. Kozlov and K. Staniek, 2003. Are mitochondria a spontaneous and permanent source of reactive oxygen species? Redox. Rep., 8: 135-141. DOI: 10.1179/135100003225001502

Northover, B.J., 1991. Effects of pretreatment with caffeine or ryanodine on the myocardial response to simulated ischaemia. Br. J. Pharmacol., 103: 1225-1229. DOI: $10.1111 /$ j.1476-5381.1991.tb12328.x
Oleynikov, D., S. Vasilieva and A. Jashin, 2015. Testosterone and myocardial extract as a correction scheme in case of experimental doxorubicin cardiomyopathy. Canad. Sci. J.

Olson, R.D. and P.S. Mushlin, 1990. Doxorubicin cardiotoxicity: Analysis of prevailing hypotheses. FASEB J., 4: 3076-3086. PMID: 2210154

Olson, R.D., P.S. Mushlin, D.E. Brenner, S. Fleischer and B.J. Cusack et al., 1988. Doxorubicin cardiotoxicity may be caused by its metabolite, doxorubicinol. Proc. Natl. Acad. Sci., 85: 3585-3589. PMID: 2897122

Opie, L.H., F.T. Thandroyen, C. Muller and O.L. Bricknell, 1979. Adrenaline-induced "oxygen-wastage" and enzyme release from working rat heart. Effects of calcium antagonism, beta-blockade, nicotinic acid and coronary artery ligation. J. Mol. Cell Cardiol., 11: 1073-1094. PMID: 522135

Opie, L.H., 2006. The Metabolic Syndrome, Does it Exist? In: Diabetes at the Limits, Opie, L.H., M. Kasuga and D.M. Yellon (Eds.), University of Cape Town Press, Cape Town, South Africa, pp: 95-110.

Osorio, J.C., W.C. Stanley, A. Linke, M. Castellari and Q.N. Diep et al., 2002. Impaired myocardial fatty acid oxidation and reduced protein expression of retinoid $\mathrm{x}$ receptor- $\alpha$ in pacing-induced heart failure. Circulation, 106: 606-612.

DOI: 10.1161/01.CIR.0000023531.22727.C1

Outomuro, D., D.R. Grana, F. Azzato and J. Milei, 2007. Adriamycin-induced myocardial toxicity: New solutions for an old problem? Int. J. Cardiol., 117: 6-15. DOI: 10.1016/j.ijcard.2006.05.005

Paolisso, G., S. De Riu, G. Marrazzo, M. Verza and M. Varricchio et al., 1991. Insulin resistance and hyperinsulinemia in patients with chronic congestive heart failure. Metabolism, 40: 972-977. DOI: 10.1016/0026-0495(91)90075-8

Paolisso, G., A. Gambardella, D. Galzerano, A. D'Amore and P. Rubino et al., 1994. Total-body and myocardial substrate oxidation in congestive heart failure. Metabolism, 43: 174-179.

DOI: 10.1016/0026-0495(94)90241-0

Paternostro, G., D. Pagano, T. Gnecchi-Ruscone, R.S. Bonser and P.G. Camici, 1999. Insulin resistance in patients with cardiac hypertrophy. Cardiovascular Res., 42: 246-253.

DOI: 10.1016/S0008-6363(98)00233-8

Pessah, I.N., M.J. Schiedt, M.A. Shalaby, M. Mack and S.N. Giri, 1992. Etiology of sarcoplasmic reticulum calcium release channel lesions in doxorubicininduced cardiomyopathy. Toxicology, 72: 189-206. DOI: 10.1016/0300-483X(92)90112-R

Pieske, B., L.S. Maier, V. Piacentino, J. Weisser and G. Hasenfuss et al., 2002. Rate dependence of $[\mathrm{Na}+] \mathrm{i}$ and contractility in nonfailing and failing human myocardium. Circulation, 106: 447-453. DOI: 10.1161/01.CIR.0000023042.50192.F4 
Randle, P.J., P.B. Garland, C.N. Hales and E.A. Newsholme, 1963. The glucose fatty-acid cycle its role in insulin sensitivity and the metabolic disturbances of diabetes mellitus. Lancet, 1: 785-789. DOI: 10.1016/S0140-6736(63)91500-9

Razeghi, P., M.E. Young, J.L. Alcorn, C.S. Moravec and O.H. Frazier et al., 2001. Metabolic gene expression in fetal and failing human heart. Circ, 104: 29232931. DOI: $10.1161 /$ hc4901.100526

Rechelt, M.E., K.M. Mellor, C.L. Curl, D. Stapleton and L.M.D. Delbridge et al., 2013. Myocardial glycophagy - A specific glycogen handling response to metabolic stress is accentuated in the female heart. J. Molecular Cellular Cardiol., 65: 67-75. DOI: 10.1016/j.yjmcc.2013.09.014

Sack, M.N., T.A. Rader, S. Park, J. Bastin and S.A. McCune et al., 1996. Fatty acid oxidation enzyme gene expression is downregulated in the failing heart. Circulation, 94: 2837-2842. PMID: 8941110

Saha, A.K., A.J. Schwarsin, R. Roduit, F. Masse and V. Kaushik et al., 2000. Activation of malonyl-CoA decarboxylase in rat skeletal muscle by contraction and the AMP-activated protein kinase activator 5aminoimidazole-4-carboxamide-1-beta -Dribofuranoside. J. Biol. Chem., 275: 24279-24283. PMID: 10854420

Schlattner, U., M. Forstner, M. Eder, O. Stachowiak and K. Fritz-Wolf et al., 1998. Functional aspects of the X-ray structure of mitochondrial creatine kinase: A molecular physiology approach. Mol. Cell Biochem., 184: 125-140.

DOI: 10.1023/A:1006851330913

Scwenk, R.W. and J.J.F.P. Luiken, 2008. Regulation of sarcolemmal glucose and fatty acid transporters in cardiac disease. Cardiovascular Res., 79: 249-258. DOI: $10.1093 / \mathrm{cvr} / \mathrm{cvn} 116$

Shanik, M.H., Y. Xu, J. Škrha, R. Dankner and Y. Zick et al., 2008. Insulin resistance and hyperinsulinemia. Diabetes Care, 31: 262-268. DOI: $10.2337 / \mathrm{dc} 08$-s264

Shearer, J., P.T. Fueger, J.N. Rottman, D.P. Bracy and B. Binas et al., 2005. Heart-type fatty acid-binding protein reciprocally regulates glucose and fatty acid utilization during exercise. Am. J. Physiol. Endocrinol. Metab, 288: E292-E297.

DOI: 10.1152/ajpendo.00287.2004

Shibayama, J., T.N. Yuzyuk, J. Cox, A.V. Zaitsev and M. Miller et al., 2015. Metabolic remodeling in moderate synchronous versus dyssynchronous pacing-induced heart failure: Integrated metabolomics and proteomics study. PLoS ONE, 10: e0118974-e0118974. PMID: 25790351

Stanley, W.C., G.D. Lopaschuk and J.G. McCormack, 1997. Regulation of energy substrate metabolism in the diabetic heart. Cardiovasc Res., 34: 25-33. PMID: 9217869
Steinberg, G.R. and B.E. Kemp, 2009. AMPK in health and disease. Physiol. Rev., 89: 1025-1078. PMID: 19584320

Stoppani, J., A.L. Hildebrandt, K. Sakamoto, D. Cameron-Smith and L.J. Goodyear et al., 2002. AMP-activated protein kinase activates transcription of the UCP3 and HKII genes in rat skeletal muscle. Am. J. Physiol. Endocrinol. Metab., 283: E1239-E1248. PMID: 12388122

Sutko, J.L. and J.A. Airey, 1996. Ryanodine receptor $\mathrm{Ca}^{2+}$ release channels: Does diversity in form equal diversity in function? Physiol. Rev., 76: 1027-1071. PMID: 8874493

Swain, S.M., F.S. Whaley and M.S. Ewer, 2003. Congestive heart failure in patients treated with doxorubicin: A retrospective analysis of three trials. Cancer, 97: 2869-2879. PMID: 12767102

Takemura, G. and H. Fujiwara, 2007. Doxorubicininduced cardiomyopathy from the cardiotoxic mechanisms to management. Prog. Cardiovasc. Dis., 49: 330-352. PMID: 17329180

Taylor, M., T.R. Wallhaus, T.R. Degrado, D.C. Russell and P. Stanko et al., 2001. An evaluation of myocardial fatty acid and glucose uptake using PET with [18F]Fluoro-6-thia-heptadecanoic acid and [18F]FDG in patients with congestive heart failure. J. Nuclear Med., 42: 55-62. PMID: 11197981

Tokarska-Schlattner, M., M. Zaugg, R. da Silva, E. Lucchinetti and M.C. Schaub et al., 2005. Acute toxicity of doxorubicin on isolated perfused heart: Response of kinases regulating energy supply. Am. J. Physiol. Heart Circul. Physiol., 289: 37-47. DOI: 10.1152/ajpheart.01057.2004

Tokarska-Schlattner, M., M. Zaugg, C. Zuppinger, T. Wallimann and U. Schlattner, 2006. New insights into doxorubicin-induced cardiotoxicity: The critical role of cellular energetics. J. Mol. Cell Cardiol., 41: 389-405. DOI: 10.1016/j.yjmcc.2006.06.009

van der Vusse, G.J., M. van Bilsen and J.F. Glatz, 2000. Cardiac fatty acid uptake and transport in health and disease. Cardiovasc Res., 45: 279-293. DOI: 10.1016/S0008-6363(99)00263-1

Ventura-Clapier, R., A. Garnier and V. Veksler, 2004. Energy metabolism in heart failure. J. Physiol., 555: 1-13. DOI: 10.1113/jphysiol.2003.055095

Von Hoff, D.D., M.W. Layard, P. Basa, H.L. Davis and A.L. Von Hoff et al., 1979. Risk factors for doxorubicin-lnduced congestive heart failure. Ann. Intern. Med., 01: 710-717. DOI: $10.7326 / 0003-4819-91-5-710$

Wallimann, T., M. Wyss, D. Brdiczka, K. Nicolay and H.M. Eppenberger, 1999. Intracellular compartmentation, structure and function of creatine kinase isoenzymes in tissues with high and fluctuating energy demands: The 'phosphocreatine circuit' for cellular energy homeostasis. Biochem. J., 281: 21-40. DOI: $10.1042 / b j 2810021$ 
Wallis, J., C.A. Lygate, A. Fischer, H.M. Ten and J.E. Schneider et al., 2005. Supranormal myocardial creatine and phosphocreatine concentrations lead to cardiac hypertrophy and heart failure: Insights from creatine transporter-overexpressing transgenic mice. Circulation, 112: 3131-3139.

DOI: 10.1161/CIRCULATIONAHA.105.572990

Wang, G.W., J.B. Klein and Y.J. Kang, 2001. Metallothionein inhibits doxorubicin-induced mitochondrial cytochrome c release and caspase- 3 activation in cardiomyocytes. J. Pharmacol. Exp. Therapeut., 298: 461-468. PMID: 11454906

Wang, W., P. Hansen, B.A. Marshall, J.O. Holloszy and M. Mueckler, 1996. Insulin unmasks a COOHterminal Glut4 epitope and increases glucose transport across T-tubules in skeletal muscle. J. Cell Biol., 135: 415-430. PMID: 8896598

Weinstein, D.M., J. Mihm and J.A. Bauer, 2000. Cardiac peroxynitrite formation and left ventricular dysfunction following doxorubicin treatment in mice. J. Pharmacol. Exp. Taltreat., 294: 396-401. PMID: 10871338

Wheeler, T.J., R.D. Fell and M.A. Hauck, 1994. Translocation of two glucose transporters in heart: Effects of rotenone, uncouplers, workload, palmitate, insulin and anoxia. Biochim. Biophys. Acta, 1196: 191-200.

DOI: $10.1016 / 0005-2736(94) 00211-8$

Wilson, J.R., P. Douglas, W.F. Hickey, V. Lanoce and N. Ferraro et al., 1987. Experimental congestive heart failure produced by rapid ventricular pacing in the dog: Cardiac effects. Circulation, 75: 857-867. DOI: $10.1161 / 01 . C I R .75 .4 .857$

Witteles, R.M., W.H.W. Tang, A.H. Jamali, J.W. Chu and G.M. Reaven et al., 2004. Insulin resistance in idiopathic dilated cardiomyopathy: A possible etiologic link. J. Am. College Cardiol., 44: 78-81. PMID: 15234411

$\mathrm{Xu}$, S., M. Galperin, G. Melvin, R. Horowits and N. Raben et al., 2010. Impaired organization and function of myofilaments in single muscle fibers from a mouse model of Pompe disease. J. Applied Physiol., 108: 1383-1388. PMID: 20223998

$\mathrm{Xu}, \mathrm{X}$., H.L. Persson and D.R. Richardson, 2005. Molecular pharmacology of the interaction of anthracyclines with iron. Molecular Pharmacol., 68: 261-271. PMID: 15883202

Yeh, E.T., A.T. Tong and D.J. Leniha, 2004. Cardiovascular complications of cancer therapy: Diagnosis, pathogenesis and management. Circulation, 109: 3122-3131. PMID: 15226229

Kim, Y.B., O.D. Peroni, W.G. Aschenbach, Y. Minokoshi and K. Kotani et al., 2005. Muscle-specific deletion of the glut4 glucose transporter alters multiple regulatory steps in glycogen metabolism. Mol. Cell Biol., 25: 9713-9723. PMID: 16227617
Zhou, S., C.M. Palmeira and K.B. Wallace, 2001a. Doxorubicin-induced persistent oxidative stress to cardiac myocytes. Toxicol. Lett., 121: 151-157. PMID: 11369469

Zhou, S., A. Starkov, M.K. Froberg, R.L. Leino and K.B. Wallace, 2001b. Cumulative and irreversible cardiac mitochondrial dysfunction induced by doxorubicin. Cancer. Res., 61: 771-777. PMID: 11212281

Zou, M.H., X.Y. Hou, C.M. Shi, S. Kirkpatick and F. Liu et al., 2003. Activation of 5'-AMP-activated kinase is mediated through c-Src and phosphoinositide 3-kinase activity during hypoxiareoxygenation of bovine aortic endothelial cells. Role of peroxynitrite. J. Biol. Chem., 278: 34003-34010. PMID: 12824177

Zucchi, R. and S. Ronca-Testoni, 1997. The sarcoplasmic reticulum $\mathrm{Ca}^{2+}$ channel/ryanodine receptor: Modulation by endogenous effectors, drugs and disease states. Pharmacol. Rev., 49: 1-51. PMID: 9085308

Zucchi, R. and R. Danesi, 2003. Cardiac toxicity of antineoplastic anthracyclines. Curr. Med. Chem. Anticancer Agents, 3: 151-71. DOI: $10.2174 / 1568011033353434$

Zucker, I.H., 2006. Novel mechanisms of sympathetic regulation in chronic heart failure. Hypertension, 48: 1005-1011.

Zuppinger, C., F. Timolati and T.M. Suter, 2007. Pathophysiology and diagnosis of cancer drug induced cardiomyopathy. Cardiovasc Toxicol., 7: 61-66. PMID: 17652805

\author{
Abbreviations \\ Acyl-coenzyme A-acyl-CoA \\ ADP-adenosine diphosphate \\ AMP-Adenosine monophosphate \\ AMPK-5' adenosine monophosphate-activated protein \\ kinase \\ ATP-adenosine triphosphate \\ CDVD-chronic degenerative valvular disease \\ CK-creatine kinase \\ DCM-dilated cardiomyopathy \\ Dox-doxorubicin \\ DoxCM-doxorubicin cardiomyopathy \\ FFA-free fatty acids \\ GLUT-glucose transporters \\ Ins-insulin \\ IR-insulin resistance \\ LDH-lactate dehydrogenase \\ PCr-phosphocreatine \\ PDH-pyruvate dehydrogenase \\ ROS-reactive oxygen species \\ TCA-tricarboxylic acid cycle
}

